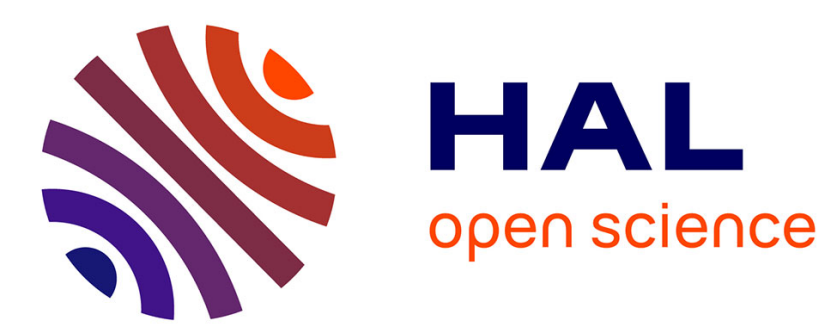

\title{
Level-Set method and stability condition for curvature-driven flows
}

Cedric Galusinski, Paul Vigneaux

\section{To cite this version:}

Cedric Galusinski, Paul Vigneaux. Level-Set method and stability condition for curvature-driven flows. Comptes rendus hebdomadaires des séances de l'Académie des sciences, 2007, 344 (11), pp.703-708. 10.1016/j.crma.2007.05.001 . hal-00193189

\section{HAL Id: hal-00193189 https://hal.science/hal-00193189}

Submitted on 1 Dec 2007

HAL is a multi-disciplinary open access archive for the deposit and dissemination of scientific research documents, whether they are published or not. The documents may come from teaching and research institutions in France or abroad, or from public or private research centers.
L'archive ouverte pluridisciplinaire HAL, est destinée au dépôt et à la diffusion de documents scientifiques de niveau recherche, publiés ou non, émanant des établissements d'enseignement et de recherche français ou étrangers, des laboratoires publics ou privés. 
Numerical Analysis

\title{
Level-Set method and stability condition for curvature-driven flows
}

\author{
Cédric Galusinski ${ }^{\mathrm{a}}$, Paul Vigneaux ${ }^{\mathrm{b}, 1}$ \\ a MC2 (INRIA Futurs) \& ANAM/CPT - Université du Sud Toulon Var, Avenue de l'Université - BP20132 83957 La \\ Garde Cedex, France \\ ${ }^{\mathrm{b}}$ MC2 (INRIA Futurs) \& MAB - Université Bordeaux 1, 351 Cours de la Libération, 33405 Talence Cedex, France
}

\begin{abstract}
We consider models for the simulation of curvature-driven incompressible bifluid flows, where the surface tension term is discretized explicitly. From this formulation a numerical stability condition arises for which we present a new theoretical estimation for low and medium Reynolds numbers. We illustrate our analysis with numerical simulations of microfluidic flows using Level Set method. Finally, we propose a method to reduce computational cost induced by this stability condition for low flow velocities. To cite this article: C. Galusinski, P. Vigneaux, C. R. Acad. Sci. Paris, Ser. I 344 (2007).
\end{abstract}

\section{Résumé}

Méthode Level Set et condition de stabilité pour des écoulements pilotés par la tension de surface. Dans cette Note, nous considérons des modèles utilisés pour simuler des écoulements bifluides incompressibles, pilotés par la tension de surface. En particulier, lorsque la discrétisation du terme de tension de surface est explicite, une condition de stabilité contraint le pas de temps et nous en proposons ici une nouvelle estimation théorique, pour des écoulements dont le nombre de Reynolds est faible ou modéré. Notre analyse est étayée par des simulations numériques, basées sur la méthode Level Set et appliquées à des écoulements en microfluidique. Nous proposons, de plus, une méthode pour réduire les coûts de calculs induits par cette condition de stabilité pour des écoulements lents. Pour citer cet article : C. Galusinski, P. Vigneaux, C. R. Acad. Sci. Paris, Ser. I 344 (2007).

Email addresses: galusins@univ-tln.fr (Cédric Galusinski), vigneaux@math.u-bordeaux1.fr (Paul Vigneaux).

1 Corresponding author

Preprint submitted to HAL 


\section{Version française abrégée}

Durant la dernière décennie, le champ de la microfluidique s'est drastiquement diversifié : il permet le transport de trés faibles volumes de fluide (nanolitres) à travers des canaux du diamètre d'un cheveu. Cette miniaturisation est de plus en plus utilisée pour construire un vaste panel d'outils dont, par exemple, l'utilisation de microgouttes comme des réacteurs chimiques. La simulation de ces écoulements soulève de nouvelles difficultés en mécanique des fluides numérique.

Dans cette Note, on s'intéresse en particulier à la simulation d'écoulements bifluides incompressibles, pilotés par la tension de surface. En suivant une approche classique pour la modélisation de tels écoulements, on utilise une formulation de suivi d'interface découplant le calcul de la vitesse de l'écoulement et le déplacement de l'interface (2) (3) (4). Ainsi, la discrétisation du terme de tension de surface est explicite. De cette formulation découle une condition de stabilité dont nous proposons ici une nouvelle estimation théorique, pour des écoulements dont le nombre de Reynolds est faible ou modéré :

Proposition 0.1 Supposons que (2) (3) (4) soit discrétisé en temps à l'aide d'une discrétisation explicite du terme de tension de surface. Alors, pour une telle discrétisation en temps et quelque soit la discrétisation en espace, le schéma numérique associé est stable sous la condition

$$
\Delta t \leq \min \left(\Delta t_{c}, \Delta t_{\sigma}\right), \text { avec } \Delta t_{c}=c_{1}\|u\|_{L^{\infty}(\Omega)}^{-1} \Delta x \text { et } \Delta t_{\sigma}=c_{2} \frac{\eta}{\sigma} \Delta x
$$

où $\Delta t$ est le pas de discrétisation en temps, $\Delta x$ est le pas de discrétisation en espace et $c_{1}, c_{2}$ ne dépendent pas des données du problème.

Ensuite, à l'aide d'une formulation Level Set pour le suivi d'interface [8], nous retrouvons numériquement cette condition de stabilité. Nous montrons de plus que son non-respect conduit à la création de courants parasites associés à des oscillations de l'interface.

Pour des écoulements « lents », cette condition de stabilité se révèle être trés restrictive. Nous proposons donc une méthode pour diminuer les temps de calculs. Ces écoulements induisent des formes d'interface stabilisées que nous exploitons pour introduire un référentiel (13) (14) où l'écoulement devient stationnaire. Les coûts de calculs sont alors drastiquement diminués car il ne reste plus qu'à translater les états stationnaires obtenus dans ce référentiel.

\section{A model for bifluid flows}

The field of microfluidics has dramatically improved over the last decade : it enables the transport of minuscule amounts of fluid (nanolitres) through channels the diameter of a single human hair. This miniaturization is increasingly employed to build a wide range of technological devices for example, genome sequencing [2] or using microdroplets as chemical reactors [4]. But a better understanding of the evolution of droplets as they move through microchannels is necessary in order to achieve the flow control needed in practical applications. Our development of a numerical model for simulating bifluid flows in microchannels leads us to the result of this Note : the derivation of a stability condition induced by surface tension when this term is discretized explicitly. Furthermore, it appears that in microchannels, due to low flow velocities, this stability condition is heavily restrictive ; we thus propose a splitting approach to relax the numerical cost. 
We consider the following model based upon the Level Set method [5] [6] proposed by Sussman et al. [8] for incompressible two-phase flows :

$$
\begin{aligned}
& \rho\left(u_{t}+u . \nabla u\right)-\operatorname{div}(2 \eta D u)+\nabla p=\sigma \kappa \delta(\phi) n \quad \forall(t, x) \in \mathbb{R}^{+} \times \Omega, \\
& \operatorname{div}(u)=0 \quad \forall(t, x) \in \mathbb{R}^{+} \times \Omega, \\
& \phi_{t}+u . \nabla \phi=0 \quad \forall(t, x) \in \mathbb{R}^{+} \times \Omega,
\end{aligned}
$$

where $\Omega$ is the $2 \mathrm{D}$ (or 3D) bounded fluid domain, $\nabla$ denotes the gradient operator with respect to the variable $x, D u=\left(\nabla u+\nabla^{T} u\right) / 2$, the density $\rho$ is assumed constant, $\eta$ is the variable viscosity such that $: \eta=\eta(\phi)=\eta_{i}, i=1,2$ in fluid $i, \sigma$ is the surface tension coefficient, $n$ is the normal to the interface, $\kappa$ is the curvature of the interface and $\delta(\phi)$ is the Dirac delta function localized on the interface. Different boundary conditions on the velocity $u$ close the system.

\section{Surface tension induced stability condition}

Because the stability condition induced by surface tension, proposed by Brackbill et al. [1] for NavierStokes equation, involves the density but not the viscosity : this condition is only valid in certain flow regimes (high Reynolds numbers). It is therefore necessary to derive another stability condition, for both low and medium Reynolds numbers. In the following, we propose a new stability condition induced by surface tension for Navier-Stokes equation which is also the stability condition for Stokes equation. This stability condition is induced by the explicit discretization of the surface tension term and avoids oscillatory behaviour of the interface around an asymptotic shape of interface. These oscillations are related to what is known in the literature as parasitic currents [7].

\subsection{Stability analysis}

Proposition 2.1 Assume that (2) (3) (4) is discretized in time by an explicit discretization of the surface tension term. Then, a numerical scheme, associated to such a time discretization and all space discretizations, is stable under the condition

$$
\Delta t \leq \min \left(\Delta t_{c}, \Delta t_{\sigma}\right), \text { with } \Delta t_{c}=c_{1}\|u\|_{L^{\infty}(\Omega)}^{-1} \Delta x \text { and } \Delta t_{\sigma}=c_{2} \frac{\eta}{\sigma} \Delta x
$$

where $\Delta t$ is the time step, $\Delta x$ is space step of the discretization, and $c_{1}, c_{2}$ do not depend on the data of the problem.

Proof : The first constraint $\Delta t \leq c_{1}\|u\|_{L^{\infty}(\Omega)}^{-1} \Delta x$ is the classical CFL condition where $c_{1}$ depends on the choice of the scheme to discretize the transport equation (4). The second condition avoids oscillations phenomena of the interface. We perform the analysis on the continuous problem instead of the discrete problem. For that, we assume that the numerical scheme approximates consistently the continuous problem. We perform a stability analysis by an interface perturbation approach. The estimate of the velocity induced by the perturbation is the key point of the analysis.

We consider a perturbation of amplitude $\delta$ and wavelength $L$ of the interface $\Gamma_{0}$.

Let us recall that in the Level Set framework, a regularization of Dirac delta function $(\nabla H(\phi))$ is obtained by considering $H_{\varepsilon}$ instead of $H$, where $H_{\varepsilon}$ is a smooth increasing function and approximates the Heaviside function as $\varepsilon$ goes to zero, $\operatorname{supp} H_{\varepsilon}^{\prime} \subset(-\varepsilon ; \varepsilon),\left\|H_{\varepsilon}^{\prime}\right\|_{\infty} \leq \frac{2}{\varepsilon}$. 
We then denote $w$ the difference between the velocity induced by the unperturbed interface $\Gamma_{0}$ and the velocity induced by the perturbed interface, $\Gamma$. The perturbation of the surface tension term, noted $f$ in the following, can be estimated, in $L^{\infty}(\Omega)$ norm, by :

$$
\|f\|_{L^{\infty}(\Omega)} \leq c \sigma\left(\frac{\delta}{L^{3}}+\left\|\partial_{\tau} \kappa_{0}\right\|_{L^{\infty}\left(\Gamma_{0}\right)} \frac{\delta}{\varepsilon}\right)
$$

where $c$ does not depend on the whole parameters, $\kappa_{0}$ is the curvature of the unperturbed problem and $\partial_{\tau}$ denotes the tangential derivative along $\Gamma_{0}$.

Assuming velocity perturbation responds, in main part, with the same frequency $L$ (which is numerically verified), we have :

$$
\|\nabla w\|_{L^{2}(\Omega)} \sim \frac{1}{L}\|w\|_{L^{2}(\Omega)}
$$

Therefore, plugging (7) in a classical energy estimate, one shows there exists a constant $C$ such that :

$$
\frac{\rho}{2} \frac{d}{d t}\|w\|_{L^{2}(\Omega)}^{2}+\left(\frac{C \eta}{L^{2}}-\rho\|\nabla u\|_{L^{\infty}(\Omega)}\right)\|w\|_{L^{2}(\Omega)}^{2} \leq \frac{L^{2}}{C \eta}\|f\|_{L^{2}(\Omega)}^{2}
$$

and, if $\rho\|\nabla u\|_{L^{\infty}(\Omega)} \leq \frac{C \eta}{2 L^{2}}$, which is true for smooth flow and sufficiently small wavelength $L$, we get :

$$
\|w\|_{L^{2}(\Omega)} \leq \frac{L^{2}}{C \eta}\|f\|_{L^{2}(\Omega)}
$$

Invoking again the profile of the solution (as for assumption (7)) and the surface tension term, we have :

$$
\|w\|_{L^{\infty}(\Omega)} \leq \frac{L^{2}}{C \eta}\|f\|_{L^{\infty}(\Omega)}
$$

An explicit time discretization of the source term obliges to consider sufficiently small time step so that the displacement (during the time step) of the interface is smaller than the size $\delta$ of the perturbation of the interface. If the displacement is bigger than $2 \delta$, perturbations are amplified and oscillates. Finally, we can write the stability condition by saying that the oscillations are removed if :

$$
\Delta t_{\sigma}=\frac{\delta}{\|w\|_{L^{\infty}(\Omega)}} \leq c^{\prime} \frac{\eta}{\sigma} \frac{L}{1+\left\|\partial_{\tau} \kappa_{0}\right\|_{L^{\infty}\left(\Gamma_{0}\right)} \frac{L^{3}}{\varepsilon}},
$$

where $c^{\prime}$ does not depend on the physical parameters. As the wavelength $L$ is upper bounded and $\varepsilon$ is chosen of size $\Delta x$, this condition is restrictive for the smaller wavelength admissible in the numerical process. We are then concerned with $L \sim \Delta x$, and it reads :

$$
\Delta t_{\sigma} \sim \frac{\eta}{\sigma} \Delta x
$$

Remark 1 If the flow velocity is very low then the previous condition on the time step is very restrictive since $\Delta t_{c} \gg \Delta t_{\sigma}$ (e.g. in microfluidics).

Remark 2 There are two ways to relax the stability constraint. First, as mentioned in the reference work of Brackbill et al., this constraint would be removed by an implicit treatment of surface tension term. A step in this direction has been done recently by Hysing [3]. Second, smoothing of the curvature is another way to relax the constraint. Indeed, following the proof, a smoothing allows the artificial increase of the capillary time step $\Delta t_{\sigma}$.

In order to reduce the numerical cost, we propose another method, which relies on the use of the steady behaviour of the interface in some zones of channels. We will detail this method in the following section. 


\section{Numerical results}

We focus on numerical simulation of microfluidic flows with very low Reynolds number.

\subsection{Numerical stability condition}

In practical applications, we need to determine the constant $c_{2}$. Running numerical tests with a Stokes code and taking a constant $\eta$, we find that $c_{2}=6$ is the critical value for which the computation is always stable when we refine the mesh. These numerical experiments show that $c_{2}=6$ is independent of the space step $\Delta x$, and $c_{2}=8$ is stable except for too fine grids. Figure 1 shows numerical simulations of an unconfined droplet in a microchannel with two values of $c_{2}$.
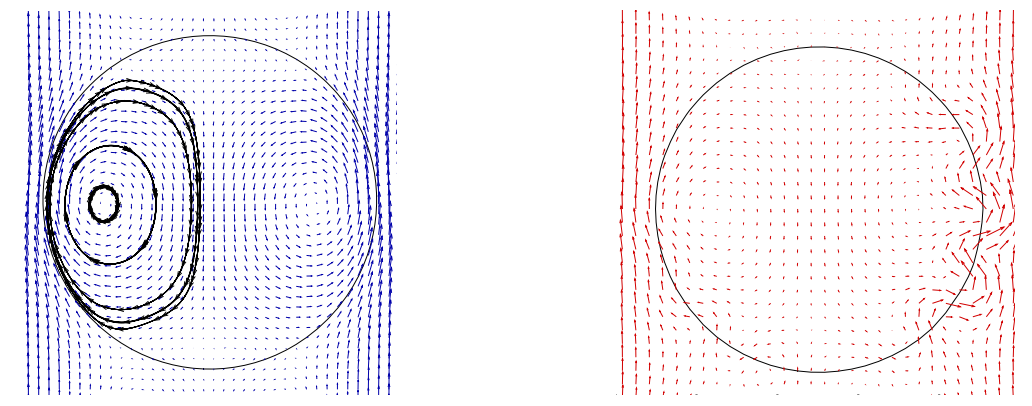

Figure 1. on the left (velocity field in blue, interface in black and some streamlines in arrowed-line), $c_{2}=8$ and the computation is stable for a medium grid, whereas on the right (velocity field in red, interface in black), $c_{2}=9$ and the instability appears on the same grid ; velocity fields are those in the drop frame of reference

\subsection{Mixer analysis: The drop frame of reference}

We want to perform numerical simulations of microdroplets to study mixing inside them by observing the streamlines in the drop frame of reference.

In straight microchannels the shape of the droplets is stabilized and we have to exploit this stationary phenomena in order to reduce the numerical cost. To this end, we work in the drop frame of reference.

\subsubsection{Find the scalar droplet's velocity}

In order to work in the drop frame of reference, we have to define the scalar droplet's velocity. This velocity has a meaning as soon as the shape of the droplet is stabilized and then there exists a scalar $u_{d}$ such that:

$$
u=u_{d} U+v, v \cdot n=0 \text { on } \Gamma_{0},
$$

where $U$ is a "unitary" flow parallel to the wall in a regular channel. Then the droplet moves with the global velocity $u_{d} U$ and the velocity in the drop frame of reference is $v$. In the general case, with stabilized or destabilized droplet shape, we define the local droplet's velocity on the fluid interface where $U \cdot n \neq 0$ as

$$
u_{d}^{l o c}=\frac{u \cdot n}{U \cdot n} \text { on } \Gamma_{0} .
$$

We then define the droplet's velocity as the mean value of local droplet velocities. 


\subsubsection{Splitting of the flow}

In straight microchannels, the velocity of a stabilized droplet verifies (13). We reduce the computational cost by using the algorithm which consists in translating the droplet while $v \cdot n$ remains close to zero. This is obtained with a standard CFL condition on the time step with velocity $u_{d} U$.

To sum up, the algorithm consists in two steps : an iterative step which allows to compute the shape modification of the interface due to surface tension (i.e. by transporting $\phi$ with $v$ using the restricting time step $\left.\Delta t_{\sigma}\right)$ and then, a step which consists in transporting $\phi$ with $u_{d} U$ using $\Delta t_{c}$. Note that this second step can be deleted if we only want to get the asymptotic shape of the droplet and not its position in the channel. Comparison of CPU time between a standard method and our splitting method depends on the length of the channel. We decrease heavily CPU time when channel's length is many times droplet's length and for low flows. More precisely, there is no theoretical saving when droplet had not reach its asymptotical shape but when droplet's shape becomes stationary and the droplet is only translating in the channel, time saving is of a factor $\Delta t_{\sigma} / \Delta t_{c}$ (which, in our microfluidic applications, can be of order 100). Figures 1 and 2 show that confinement and injection velocity (among others, which will be presented
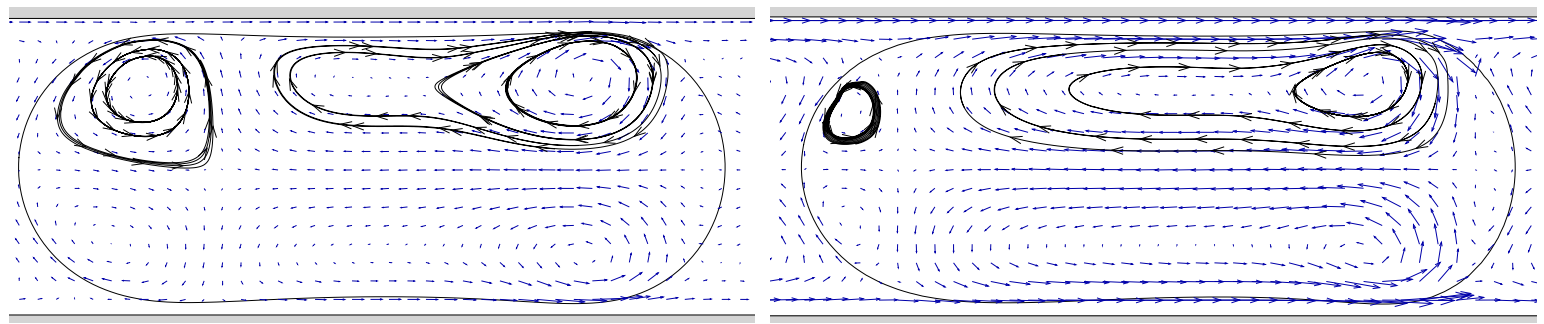

Figure 2. Various regimes for a confined droplet in a microchannel (wall in grey, velocity field in blue, interface in black and some streamlines in arrowed-line) : on the left, injection velocity is lower than on the right.

in a subsequent publication) allow to induce various mixing regimes inside droplets.

\section{Acknowledgements}

This work was partially supported by ACI-NIM "Calculs de micro-fluides"(CNRS).

\section{References}

[1] J. U. Brackbill, D. B. Kothe, C. Zemach, A continuum method for modeling surface tension, J. Comput. Phys. 100 (1992) 335-354.

[2] A. J. deMello, DNA amplification moves on, Nature 422 (06 Mar 2003) 28-29.

[3] S. Hysing, A new implicit surface tension implementation for interfacial flows, Int. J. Numer. Meth. Fluids 51 (30 June 2006) 659-672.

[4] M. Joanicot, A. Adjari, Droplet Control for Microfluidics, Science 309 (5 Aug 2005) 887-888.

[5] S. Osher, R. Fedkiw, Level Set Methods and Dynamic Implicit Interfaces, $1^{\text {st }}$ Edition, Applied Mathematical Sciences, Vol. 153, Springer, 2003.

[6] J. A. Sethian, Level Set Methods and Fast Marching Methods - Evolving interfaces in computational geometry, fluid mechanics, computer vision and materials science, $2^{\text {nd }}$ Edition, Cambridge University Press, 1999.

[7] S. Shin, S.I. Abdel-Khalik, V. Daru, D. Juric, Accurate representation of surface tension using the level contour reconstruction method, J. Comput. Phys. 203 (2005) 493-516 
[8] M. Sussman, P. Smereka, S. Osher. A level set approach for computing solutions to incompressible two-phase flow, $J$. Comput. Phys. 114 (1994) 146-159. 